\title{
Eigenmode stability analysis of drift-mirror modes in nonuniform plasmas
}

\author{
D. Yu. Klimushkin ${ }^{1}$ and L. Chen ${ }^{2,3}$ \\ ${ }^{1}$ Institute of Solar-Terrestrial Physics, Russian Academy of Sciences, Irkutsk, P.O. Box 4026, 664033, Russia \\ ${ }^{2}$ Department of Physics and Astronomy, University of California Irvine, Irvine, CA 92697-4575, USA \\ ${ }^{3}$ Institute for Fusion Theory and Simulation, Zhejiang University, Hangzhou, China
}

Received: 19 June 2006 - Revised: 9 September 2006 - Accepted: 12 September 2006 - Published: 20 October 2006

\begin{abstract}
Drift-mirror modes in a one-dimensional inhomogeneous model of the magnetosphere are studied by employing gyrokinetics, taking into account finite Larmor radius effects. A wave equation is derived which describes both the spatial structure of the modes, and its eigenvalue yields a growth rate of the mode. The finite Larmor radius effects are shown to raise the instability threshold especially for high- $m$ waves, and lead to wave propagation across field lines.
\end{abstract}

Keywords. Magnetospheric physics (Magnetosheath; MHD waves and instabilities) - Space plasma physics (Kinetic and MHD theory)

\section{Introduction}

Generic properties of the circumterrestrial plasma are its inhomogeneity, high pressure (the plasma-to-magnetic pressure ratio $\beta \sim 1$ ), and anisotropy. Under these circumstances, drift-mirror compressional waves can be collectively excited in the plasma (Hasegawa, 1969), provided that the mirror instability criterion is satisfied. From the observational point of view, drift mirror modes are often identified with compressional storm time Pc5 geomagnetic pulsations (Barfield and McPherron, 1978) and some kinds of magnetosheath modes (Narita and Glassmeier, 2005).

A realistic interpretation of drift-mirror modes must take into account finite Larmor radius (FLR) effects because these effects enter into wave equations through the combination $k_{\perp} \rho$ (here $k_{\perp}$ is a transverse to the magnetic field component of the wave vector and $\rho$ is a particles' Larmor radius), and $k_{\perp}$ is proportional to the azimuthal wave number $m$, which is considered to be large, $m \gg 1$. The compressional storm time Pc5 pulsations are characterized by high

Correspondence to: D. Yu. Klimushkin

(klimush@iszf.irk.ru) $m$ values. Besides, these waves often have small parallel wavelengths (Takahashi et al., 1987), which implies that finite corrections of $k_{\|} / k_{\perp}$ should also be taken into account. Due to FLR effects, the wave frequency $\omega$ turns out to depend on $k_{\perp}$ (Hasegawa, 1969; Pokhotelov et al., 2004). But in inhomogeneous plasma $k_{\perp}$ should, in principle, be considered as an operator, and in the WKB approximation, as a function of coordinates. Thus, studies of instabilities of inhomogeneous plasma and mode spatial structure are intimately linked with each other. These were conducted by Vetoulis and Chen (1994, 1996) and Klimushkin (2000) for Alfvén waves, and by Crabtree and Chen (2004) for compressional waves due to trapped ions. For drift-mirror waves, such a nonlocal eigenmode stability analysis has not been carried out to our best knowledge. It is especially timely now, since the CLUSTER mission makes it possibile to study the smallscale structure of ULF waves. The study of this issue is the prime objective of the present paper.

\section{The model and the main equations}

In the model under consideration, the field lines of the ambient magnetic field $B$ are supposed to have constant curvature (cylindrical model; e.g. Pokhotelov et al., 1986). All plasma parameters vary only across magnetic surfaces. The plasmato-magnetic pressure ratio is $\beta \sim 1$, but a cold electron population present in the plasma provides a shorting out of the parallel electric field $\left(E_{\|}=0\right)$. The equilibrium distribution function $F$ is assumed to be bi-Maxwellian, and the thermal velocities along and transverse to the magnetic field are $V_{\|}$ and $V_{\perp}$, respectively. Longitudinal and transverse pressures are $P_{\|}$and $P_{\perp}$, and the particles concentration is $n$. Plasma temperature is taken to be uniform.

The perturbed quantities depend on space and time as $\exp \left[-i \omega t+i k_{y} y+i \int k_{r}(r) d r+i k_{\|} l_{\|}\right]$, where $r$ is a radial coordinate (field lines' curvature radius), $y$ and $l_{\|}$are,

Published by Copernicus GmbH on behalf of the European Geosciences Union. 
consequently, the azimuthal and longitudinal coordinates, $k_{r}$ is a wave vector radial component determined from the WKB ansatz.

We start from the perpendicular plasma force balance equation (Pokhotelov et al., 2000)

$\delta P_{\perp}+\frac{B \delta B_{\|}}{4 \pi}=\frac{k_{\|}^{2}}{k_{\perp}^{2}}\left[\frac{\omega^{2}}{k_{\|}^{2} v_{A}^{2}}-1-\frac{\beta_{\perp}-\beta_{\|}}{2}\right] \frac{B \delta B_{\|}}{4 \pi}$,

where $k_{\perp}^{2}=k_{r}^{2}(r)+k_{y}^{2}$. Later on, we will neglect the term $\omega^{2} / k_{\|}^{2} v_{A}^{2}$ because we consider much lower frequencies. In also neglecting the coupling between the compressional and transverse Alfvén mode, this equation becomes

$L_{M} b=0$.

Here, $b=\omega \delta B_{\|} / c$. The operator

$L_{M}=k_{\perp}^{2}\left(\tau+a_{M}\right)+k_{\|}^{2}\left(1+\frac{\beta_{\perp}-\beta_{\|}}{2}\right)$

is a compressional (mirror) mode operator, where

$$
\begin{aligned}
& \tau= 1+\frac{4 \pi}{c^{2}} 2 \pi \sum_{e, i} \frac{q^{2}}{m} \\
& \times \int d v_{\|} d v_{\perp} v_{\perp}^{3} J_{1}^{2}\left(\frac{k_{\perp} v_{\perp}}{\omega_{c}}\right)\left(\frac{1}{V_{\perp}^{2}}-\frac{1}{V_{\|}^{2}}\right) F, \\
& a_{M}=\frac{4 \pi \omega^{2}}{c^{2}} \frac{1}{k_{\|} k_{\perp}^{2}} 2 \pi \sum_{e, i} \frac{q^{2}}{m} \int d v_{\|} d v_{\perp} v_{\perp}^{3} J_{1}^{2}\left(\frac{k_{\perp} v_{\perp}}{\omega_{c}}\right) \\
& \quad \times\left(v_{\|}-\frac{\omega-\omega_{d}}{k_{\|}}\right)^{-1}\left(\frac{\partial F}{\partial \varepsilon}+\frac{k_{y} F^{\prime}}{\omega \omega_{c}}\right) .
\end{aligned}
$$

The prime means a differentiating with respect to the radius, $r ; \omega_{c}$ is the gyrofrequency, and

$$
\omega_{d}=\frac{k_{y}}{\omega_{c}}\left(\frac{B^{\prime}}{2 B} v_{\perp}^{2}-\frac{v_{\|}^{2}}{r}\right)
$$

is the drift frequency in the inhomogeneous magnetic field. The Eq. (1) could be readily derived using the linear gyrokinetic equations (Antonsen and Lane, 1980; Catto et al., 1981; Chen and Hasegawa, 1991).

First, we consider the "classical" drift-mirror mode, that is a mode in limits $k_{\perp} \rho \rightarrow 0, k_{\|}^{2} / k_{\perp}^{2} \rightarrow 0$, and $\left|\omega-\omega_{d}\right| \ll k_{\|} V_{\|}$. We obtain from Eq. (1):

$$
\tau_{0}-\frac{8 \pi n}{B^{2}} \sum_{e, i} m V_{\perp}^{2} \frac{T_{\perp}}{T_{\|}} i \sqrt{\frac{\pi}{2}} \frac{\omega-\omega_{*}}{k_{\|} V_{\|}}=0 .
$$

Here we have denoted

$$
\tau_{0}=1+\frac{8 \pi n}{B^{2}} \sum_{e, i} m V_{\perp}^{2}\left(1-\frac{T_{\perp}}{T_{\|}}\right),
$$

$\omega_{*}=k_{y} V_{\|}^{2} \frac{(\log n)^{\prime}+3 \alpha(\log B)^{\prime}}{\omega_{c}}$.

Later on, we will neglect the pressure of the electron component. We then readily cover the following well-known relations (Hasegawa, 1969):

$\operatorname{Re} \omega=\omega_{*}$,

$\operatorname{Im} \omega=\gamma_{M} \equiv-\tau_{0} \sqrt{\frac{2}{\pi}} \frac{k_{\|} V_{\|}}{\beta_{\perp}} \frac{T_{\|}}{T_{\perp}}$,

where $\beta_{\perp}=8 \pi n m_{i} V_{\perp}^{2} / B^{2}$. These expressions describe a wave with a drift frequency $\omega_{*}$ which grows if the mirror instability criterion $\tau_{0}<0$ is satisfied.

Now we consider small but finite values of $k_{\perp} \rho$, where $\rho$ is ion Larmor radius. Also, we are going to retain the $k_{\|}^{2} / k_{\perp}^{2}$ corrections. Let us introduce the designations

$\alpha=\frac{3}{2} \beta_{\perp}\left(\frac{T_{\perp}}{T_{\|}}-1\right), \quad \delta=\frac{k_{\|}^{2}}{k_{y}^{2}}\left(1+\frac{\beta_{\perp}-\beta_{\|}}{2}\right)$,

$\omega_{t}=\sqrt{\frac{2}{\pi}} \frac{k_{\|} V_{\|}}{\beta_{\perp}} \frac{T_{\|}}{T_{\perp}}, \quad \tilde{\tau}=\tau_{0}+\alpha k_{y}^{2} \rho^{2}, \quad h=i \frac{\omega-\omega_{*}(r)}{\omega_{t}(r)}$.

Then, instead of Eq. (5), we obtain the following driftmirror mode dispersion relation:

$k_{\perp}^{2}\left[\tilde{\tau}(r)+\alpha k_{r}^{2} \rho^{2}-h(\omega, r)\right]+k_{y}^{2} \delta=0$.

This expression can be obtained from Eq. (35) of Hasegawa (1969), if we let $k_{\perp} \rho \ll 1$ there.

\section{Drift-mirror eigenmode stability analysis}

We are going to consider modes trapped across the magnetic shells. This means that the radial mode width depends on the macroscopic scale length and, thus $\left|k_{r}\right| \ll\left|k_{y}\right|$. Then Eq. (10) further reduces to

$k_{r}^{2} \alpha \rho^{2}+[\tilde{\tau}(r)-h(\omega, r)+\delta]=0$.

This equation can be written in the form

$\alpha(r) k_{r}^{2} \rho^{2}-i \frac{\omega-\omega_{0}(r)}{\omega_{t}(r)}=0$,

where $\omega_{0}$ is the solution in the $k_{r} \rho=0$ case determined by the equality

$\tilde{\tau}(r)-h\left(\omega_{0}, r\right)+\delta=0$.

The solution of this equation is $\omega_{0}=\omega_{*}(r)+i \gamma_{M}(r)$, where

$\gamma_{M}=-(\tilde{\tau}+\delta) \omega_{t}$.

Because all of the quantities in Eq. (12) are functions of the radial coordinate $r$, the radial wave vector $k_{r}$ must also depend on $r$. Hence, Eq. (12) should be considered as an equation in the WKB approximation which determines the 
function $k_{r}=k_{r}(\omega, r)$. Thus, the drift-mirror instability constitutes an eigenmode stability analysis. To determine the eigenvalue $\omega$, we need to derive the corresponding eigenmode equation and impose the appropriate boundary conditions. The simplest approach is replacing $k_{r}$ by the differential operator $-i d / d r$; and we obtain

$\rho^{2} \alpha(r) \frac{d^{2} f}{d r^{2}}+i \frac{\omega-\omega_{0}(r)}{\omega_{t}} f(r)=0$.

Here $f(r)$ is the wave function. Certainly, any equation which differs from this one with the term like $d f / d r$ yields the same leading order WKB approximation, Eq. (12), as Eq. (13) does. But these terms do not affect the eigenvalues $\omega$ in the large $k_{y}$ asymptotic limit, so it is sufficient to restrict the consideration to Eq. (13). A natural boundary condition to this equation in the coordinate $r$ is the condition that $|f|$ decays away from the localization region.

Then, we will consider the mode localized near the surface $r_{m}$, where the function $\omega_{0}(r)$ takes its maximum value. Near this point we can use an expansion

$\omega_{0}(r)=\omega_{0 m}+(1 / 2) \omega_{0}^{\prime \prime} x^{2}$,

where $x=r-r_{m}$, and the $m$-index means a function value in the point $r_{m}$. Besides, we will consider the case $|\gamma| \ll\left|\omega_{*}\right|$. Then,

$\omega_{0}^{\prime \prime} \simeq-\frac{\left|\omega_{* m}\right|}{l^{2}}$,

where $l$ is a characteristic scale of the variation of the drift frequency across magnetic shells. Denoting

$E=\frac{\omega-\omega_{0 m}}{\omega_{t m} \rho_{m}^{2} \alpha_{m}}$,

Equation (13) becomes

$\frac{d^{2} f}{d x^{2}}+\left[i E-\frac{i}{2} \frac{\left|\omega_{*}\right|}{\omega_{t} \alpha(\rho l)^{2}} x^{2}\right] f=0$

The boundary condition, meanwhile, is

$|f(x \rightarrow \pm \infty)| \rightarrow 0$,

or $|f|$ vanishes as $|x| \rightarrow \infty$. From Eq. (14) and below, the subscript " $m$ " is omitted, assuming all plasma equilibrium parameters are evaluated at the point $r_{m}$.

As we see, Eq. (14) has the same form as the Schrödinger equation for the harmonic oscillator, and value $E$ plays the role of energy. The solution of this equation is written as

$f(x)=H_{n}\left(\frac{x}{\lambda}\right) \exp \left(-\frac{x^{2}}{2 \lambda^{2}}\right)$,

where $H_{n}$ are Hermitian polynomials, $n=0,1,2, \ldots$, and $\lambda$ is a characteristic radial wavelength determined by the condition

$\frac{i}{2} \lambda^{4} \frac{\left|\omega_{*}\right|}{\omega_{t} \alpha(\rho l)^{2}}=1$.
To determine the proper root of $\lambda^{4}$, we impose the boundary condition (15). Hence, it follows that $\operatorname{Re} \lambda^{-2}>0$, that is

$\lambda^{2}=e^{-i \pi / 4}\left|\frac{2 \omega_{t} \alpha}{\omega_{*}}\right|^{1 / 2} \rho l$.

In terms of order of magnitude, $\lambda \sim O(\sqrt{\rho l})$.

The quantization condition on the eigenvalue is $i E \lambda^{2}=2 n+1$, hence

$\operatorname{Re} \omega_{n}=\omega_{*}+\left(n+\frac{1}{2}\right) \frac{\rho}{l} \sqrt{\alpha \omega_{t}\left|\omega_{*}\right|}$,

$\gamma_{n}=\operatorname{Im} \omega_{n}=\gamma_{M}-\left(n+\frac{1}{2}\right) \frac{\rho}{l} \sqrt{\alpha \omega_{t}\left|\omega_{*}\right|}$.

Following Hasegawa (1969), let us introduce the parameter

$\Delta=\frac{3}{4}\left[\beta_{\perp}\left(\frac{T_{\perp}}{T_{\|}}-1\right)-1\right]$,

representing the measure of the overshooting of the instability condition. The growth rate takes a maximum value when $\partial \gamma_{M} / \partial k_{\|}=0, \partial \gamma_{M} / \partial k_{y}=0$. Thus, $\gamma$ peaks at

$k_{y}^{*}=\frac{1}{\rho} \frac{\sqrt{2}}{3}\left(\frac{\Delta}{\frac{4 \Delta}{3}+1}\right)^{1 / 2}, \quad \delta^{*}=\frac{\Delta}{3}$,

$k_{\|}^{*}=\sqrt{\frac{2}{27}} \frac{\Delta \Upsilon}{\rho}$,

where

$\Upsilon=\left(1+\frac{\beta_{\perp}-\beta_{\|}}{2}\right)^{-1 / 2}\left(\frac{4 \Delta}{3}+1\right)^{-1 / 2}$.

Both $k_{y}^{*}$ and $k_{\|}^{*}$ are real when $\Delta>0$. Taking maximizing values of $k_{y}, \delta$, we find

$\gamma_{M}^{*}=\frac{2}{\sqrt{27 \pi}} \frac{V_{\|} T_{\|}}{\beta_{\perp} T_{\perp}} \frac{2}{3 \rho}|\Delta| \Delta \Upsilon$.

We see that the condition for $\gamma$ to be positive is still $\Delta>0$, as in the case when FLR and $k_{\|} / k_{y}$ terms are neglected. In order of magnitude,

$\gamma_{M}^{*} \sim \Delta^{2} \frac{V_{\perp}}{\rho}$.

So, as the Larmor radius increases, the growth rate decreases, but remains positive. However, according to Eq. (19), when the radial mode number $n$ becomes large, the instability is stabilized. The critical value is

$n_{c} \sim\left(\frac{L}{\rho}\right)^{3 / 2} \Delta^{5 / 4}$,

i.e. FLR effects are favorable for lowering the stabilizing $n$ number. 


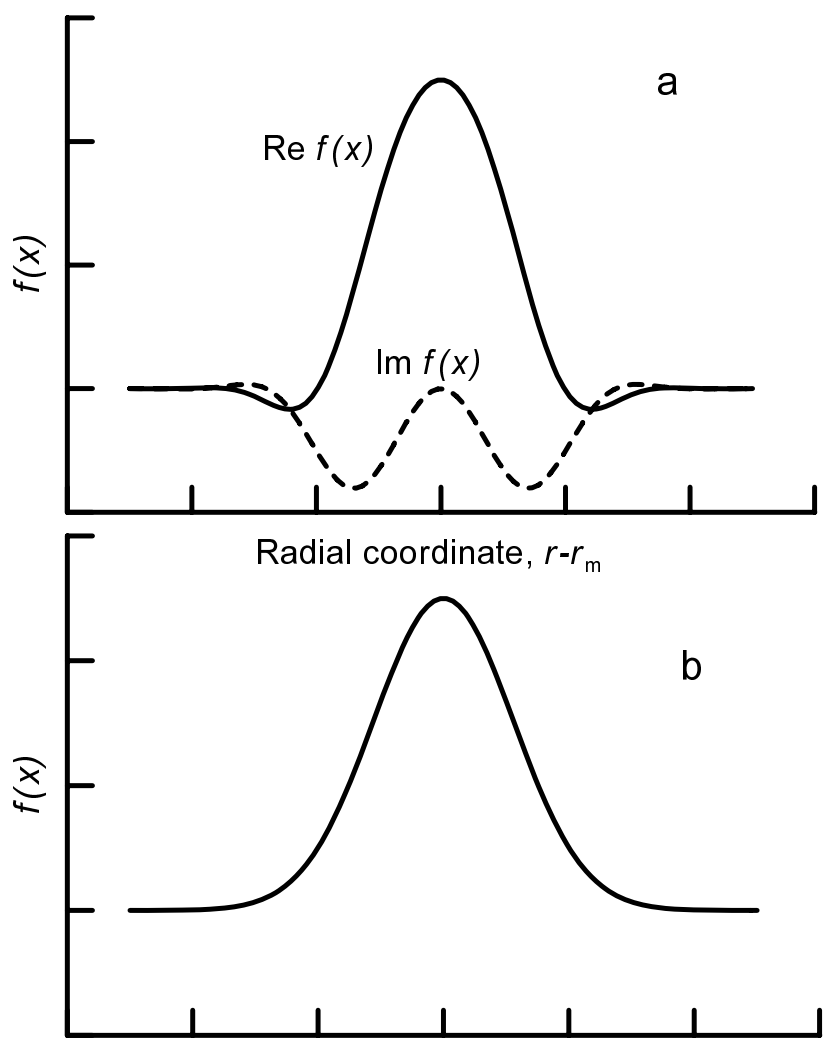

Radial coordinate, $r-r_{\mathrm{m}}$

Fig. 1. The mode structure across magnetic shells: (a) when $|\gamma| \ll\left|\omega_{*}\right|$, (b) when $|\gamma| \gg\left|\omega_{*}\right|$.

Considering the marginal instability case when $0<\Delta \ll 1$ and $k_{\|}=k_{\|}^{*}, k_{y}=k_{y}^{*}$, the characteristic radial wavelength scales are

$\lambda^{*} \sim L\left(\frac{\rho}{L}\right)^{1 / 4} \Delta^{1 / 8}$

where $L$ is the inhomogeneity scale. We see that $\lambda^{*}$ only weakly depends on both $\rho / L$ and $\Delta$.

\section{Discussions}

In this paper, we have analyzed the growth rate and the spatial structure of the drift-mirror modes as a nonlocal eigenmode problem. The following picture is beginning to emerge. At the first sight the finite Larmor radius favors the instability stabilization because the condition for the inequality becomes

$\tau_{0}+\alpha\left(k_{y} \rho\right)^{2}+\left(k_{\|} / k_{\perp}\right)^{2}\left[1+\frac{1}{2}\left(\beta_{\perp}-\beta_{\|}\right)\right]<0$.

However, at the maximizing values of $k_{y}$ and $k_{\|}$, the instability criterion remains as in the $k_{y} \rho=0$ case: $\Delta>0$. FLR effects influence the instability in another way, via lowering the threshold value of the radial harmonic number $n$. This value $n_{c}$ decreases rapidly with increasing $\rho$, and the harmonics with $n>n_{c}$ are stable.

The radial structure of the mode is described by Eq. (16). The presence of the imaginary part leads to the propagation of the wave across the magnetic shells. The real part of the radial wave vector is $\operatorname{Re} k_{r}=-x\left|\lambda^{-2}\right| \sin (\pi / 4)$, i.e. the phase velocity is directed toward the left when $r>r_{m}$ and toward the right when $r<r_{m}$. As Eq. (17) indicates, $k_{r}$ does not depend on $\omega$, i.e. energy is not transmitted across magnetic shells (like in an Alfvén wave in a homogeneous plasma). Notice that the radial structure has the oscillatory character even at $n=0$ (Fig. 1a). The wave is modulated by the Gaussian function. Surprisingly, the value of the characteristic radial wavelength has a rather weak dependence on the ratio $\rho / L$ and on the measure of the overshooting of the instability condition $\Delta$.

The situation is entirely analogous in the regions where the function $\omega_{*}(r)$ reaches minima, except that the sign of the radial phase velocity is opposite.

The conclusions about the instability remain valid when $|\gamma| \gg\left|\omega_{*}\right|$. But in this case $\operatorname{Im} \lambda^{2}=0$, i.e. the mode is standing across magnetic surfaces (Fig. 1b).

The spatial structures predicted in this paper could be compared with the CLUSTER satellite observations in the magnetosphere and in the magnetosheath.

Acknowledgements. The authors are grateful to C. Crabtree for the numerous discussions. This work was performed during D. Yu. Klimushkin's stay in University of California at Irvine. He is grateful to the staff of the Department of Physics and Astronomy of the University for the hospitality during this visit. The work of D. Yu. Klimushkin is partially supported by Russian Foundation for Basic Research grant 04-05-64321 and Program of presidium of Russian Academy of Sciences \#16 and OFN RAS \#16. L. Chen is supported by U.S.D.O.E. grant DE-FG03-94ER54736, NSF grant ATM-033527, and Guomgbiao Foundation of Zhejiang University.

Topical Editor I. A. Daglis thanks K.-H. Glassmeier for his help in evaluating this paper.

\section{References}

Antonsen, T. M. and Lane, B.: Kinetic equations for low frequency instabilities in inhomogeneous plasma, Phys. Pluids, 23, 12051214, 1981.

Barfield, J. N. and McPherron, R. L.: Storm time Pc5 magnetic pulsations observed at synchronous orbit and their correlation with the partial ring current, J. Geophys. Res., 83, 739-743, 1978.

Catto, P. J., Tang, W. M., and Baldwin, D. E.: Generalized gyrokinetics, Plasma Phys., 23, 639-650, 1981.

Chen, L. and Hasegawa, A.: Kinetic theory of geomagnetic pulsations, 1, Internal excitations by energetic particles, J. Geophys. Res., 96, 1503-1512, 1991.

Crabtree, C. and Chen, L.: Finite gyroradius theory of drift compressional modes, Geophys. Res. Lett., 31, L17804, doi:10.1029/2004GL020660, 2004. 
Hasegawa, A.: Drift mirror instability in the magnetosphere, Phys. Pluids, 12, 2642-2650, 1969.

Klimushkin, D. Yu.: The propagation of high- $m$ Alfvén waves in the Earth's magnetosphere and their interaction with high-energy particles, J. Geophys. Res., 105, 23 303-23 310, 2000.

Narita, Y. and Glassmeier, K.-H.: Dispersion analysis of lowfrequency waves through the terrestrial bow shock, J. Geophys. Res., 110, A12215, doi:10.1029/2005JA011256, 2005.

Pokhotelov, O. A., Pilipenko, V. A., Nezlina, Yu. M., Woch, J., Kremser, G., Korth, A., and Amata, E.: Excitation of high-beta plasma instabilities at the geostationary orbit: theory and observations, Planet. Space Sci., 34, 695-712, 1986.

Pokhotelov, O. A., Balikhin, M. A., Alleyne, H. S.-C. K., and Onishchenko, O. G.: Mirror instability with finite electron temperature effects, J. Geophys. Res., 105, 2393, doi:10.1029/1999JA900351, 2000.
Pokhotelov, O. A., Sagdeev, R. Z., Balikhin, M. A., and Treumann, R. A.: Mirror instability at finite ion-Larmor radius wavelengths, J. Geophys. Res., 109, A09213, doi:10.1029/2004JA010568, 2004.

Takahashi, K., Fennell, J. F., Amata, E., and Higbie, F. R.: Fieldaligned structure of the storm-time Pc5 event of November 1415, 1979, J. Geophys. Res., 92, 5857-5864, 1987.

Vetoulis, G. and Chen, L.: Global structures of Alfvén-ballooning modes in magnetospheric plasmas, Geophys. Res. Lett., 21, 2091-2094, 1994.

Vetoulis, G. and Chen, L.: Kinetic theory of geomagnetic pulsations, 3, Global analysis of drift Alfvén-ballooning modes, J. Geophys. Res., 101, 15 441-15 456, 1996. 